\title{
Influence de la séquence d'empilement et de la géométrie d'un patch composite sur l'efficacité du renforcement d'une structure métallique trouée
}

\author{
Jean-Denis Mathias ${ }^{a}$, Xavier Balandraud et Michel Grediac \\ Laboratoire de mécanique et ingénieries (LaMI), Campus de Clermont-Ferrand, Les Cézeaux, BP 265, IFMA, \\ 63175 Aubière Cedex, France
}

Reçu le 31 mars 2004, accepté le 17 octobre 2005

\begin{abstract}
Résumé - Le travail traite de l'utilisation de patchs composites pour le renforcement des structures métalliques. De nombreuses études ont été menées dans le passé sur la réparation des structures vieillissantes déjà fissurées, notamment dans le domaine de l'aéronautique. La voie étudiée ici consiste à prévenir l'apparition des fissures en collant un patch composite sur la zone à soulager ou dans son voisinage. Par analogie aux solutions adoptées en réparation, une première approche du renforcement étudiée ici consiste à coller un patch de forme carrée. Ensuite, une liberté est donnée à la géométrie du patch. Une courbe spline est choisie afin de modéliser son périmètre. Un algorithme génétique est utilisé pour optimiser les positions des points de contrôle de la courbe, ainsi que l'orientation des plis du composite. L'optimisation est appliquée dans le cas d'un essai de traction sur une plaque trouée. La solution permet une déviation des contraintes loin de la zone sensible et montre l'intérêt d'un patch de géométrie optimisée par rapport à un patch de forme carrée.
\end{abstract}

Mots clés : Patch composite / renforcement / optimisation / algorithme génétique / spline

\begin{abstract}
Influence of ply orientations and patch geometry on the efficiency of reinforcement of a metallic structure. This paper deals with the use of composite patches to relieve a loaded metallic structure. Repair of aging structure was previously studied, mainly for aircraft industry. Another solution consists in preventing the apparition of cracks or damages by bonding a composite patch on the sensible area. From solutions proposed for structure repairs, a composite patch with square shape is first tested. Then some degrees of freedom are added to the patch shape so as to strongly deviate the stresses far from the area to relieve. A spline curve is chosen to model the patch shape to reach this goal. A genetic algorithm is applied to optimize the location of the spline control points and the composite ply angles. An optimization is performed for a tensile test on a plate with a hole. It is shown that optimized patches are more efficient than square ones.
\end{abstract}

Key words: Composite patch / reinforcement / optimization / genetic algorithm / spline

\section{Introduction}

Des patchs en matériaux composites sont classiquement utilisés dans l'aéronautique pour la réparation des structures métalliques présentant des dommages de type fissures, criques ou impacts [1-5]. Une alternative à la réparation de ces structures consiste à les renforcer préventivement, avant même que les défauts n'apparaissent. Le contexte du présent travail est celui de la maintenance préventive de structures métalliques par renforts composites dans le but de retarder l'apparition de

\footnotetext{
a Auteur correspondant : jean-denis.mathias@ifma.fr
}

fissures. Une diminution de quelques pour-cent des niveaux de contraintes dans les zones à renforcer augmente en effet considérablement la durée de vie de la structure.

Par analogie aux solutions adoptées en réparation, le renforcement est effectué par collage d'un patch composite au voisinage de la zone à renforcer. L'objectif du présent travail est d'étudier l'influence de la forme du patch sur l'efficacité du renforcement.

Dans la section 2, une première approche du renforcement est effectuée à l'aide d'un patch de forme carrée entourant la zone à soulager. Le problème mécanique est résolu par l'intermédiaire du code de calcul par éléments 
finis Ansys 7.0. Cette forme carrée est classiquement utilisée en réparation pour le pontage des fissures. La performance d'une telle géométrie est estimée dans le cas du renforcement d'une plaque trouée soumise à une sollicitation de traction.

Dans la section 3, le périmètre du patch est défini par une courbe spline afin de donner plus de liberté à la géométrie du renfort. Le but est alors d'optimiser la position des points de contrôle de la courbe spline pour réduire le plus possible le niveau de contrainte dans une zone à soulager. Un autre paramètre est bien sûr les angles des plis qui constituent la stratification. Un programme d'optimisation de patchs par algorithme génétique est écrit. Ce principe d'optimisation a été développé avec succès dans divers domaines dont notamment l'optimisation de dimensionnement de stratifiés [6,7]. Il est ici étendu à l'optimisation de la géométrie du renfort.

Dans la section 4, l'algorithme génétique est appliqué dans le cas d'un essai de traction sur une plaque trouée. L'intérêt d'un patch en forme de spline sur le renforcement est ensuite examiné.

\section{Renforcement par un patch composite de forme carrée}

\subsection{Démarche issue de la réparation par patchs composites}

La réparation par patchs composites est traditionnellement utilisée pour ponter des fissures, par exemple dans les structures aéronautiques. De nombreuses recherches sur l'utilisation de patchs rivetés ou collés ont été effectuées sur ce sujet [8-10]. Ces études ont montré que le collage des patchs est préférable car les patchs rivetés entraînent de fortes concentrations de contraintes vers les trous percés pour le passage des rivets.

Le renforcement par patchs composites est une alternative à la réparation. Il doit s'effectuer avant l'apparition des fissures afin d'augmenter la durée de vie de la structure. Cet article sur le renforcement s'inscrit dans le prolongement des études sur la réparation. De ce fait, l'emploi de patchs collés est préféré à celui de patchs rivetés ou boulonnés. Dans la continuité des solutions utilisées en réparation [8-10], un patch de forme carrée est étudié pour cette première étude.

Dans le cas de la réparation, le facteur d'intensité de contrainte critique sert de critère pour évaluer l'efficacité du patch $[8,10]$. Dans le cas du renforcement, un autre critère doit être défini pour évaluer la performance du renfort. Une diminution sensible des contraintes augmente considérablement la durée de vie des structures qui sont soumises à de la fatigue. Pour cette première approche, la contrainte moyenne de Von Mises dans la zone à soulager est ici choisie comme estimateur de la performance du patch. Il s'agit d'une première étape simplificatrice, l'objectif étant à terme d'intégrer des critères de fatigue.

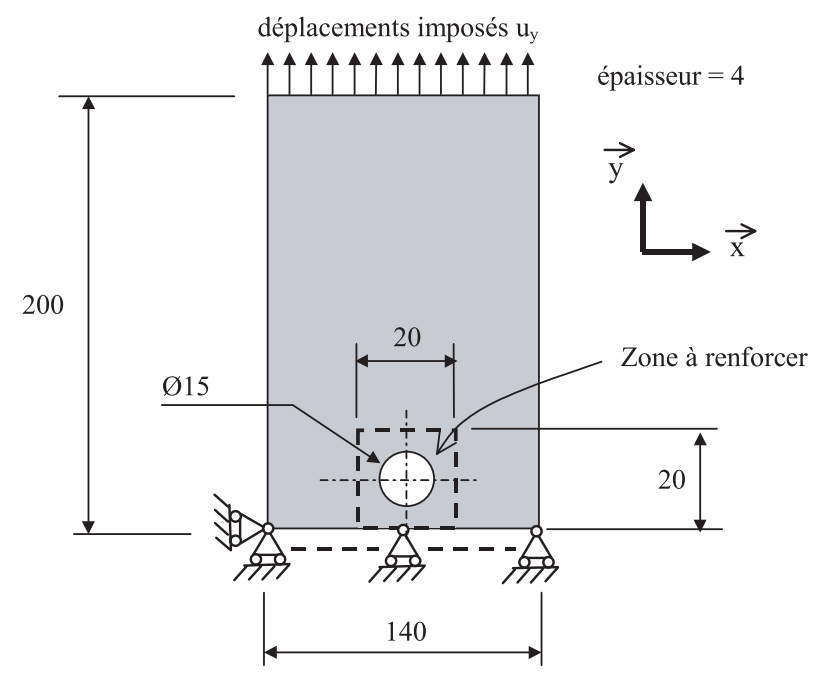

Fig. 1. Présentation du problème de renforcement.

\subsection{Présentation d'un problème de renforcement}

La structure à renforcer consiste en une plaque rectangulaire de dimensions $200 \mathrm{~mm} \times 140 \mathrm{~mm}$ avec une épaisseur de $4 \mathrm{~mm}$. Elle est en aluminium et son comportement est supposé élastique linéaire isotrope avec les caractéristiques suivantes : $E=70 \mathrm{GPa}$ et $\nu=0,3$. Cette plaque présente un trou de diamètre $15 \mathrm{~mm}$, situé près d'un bord où sont imposées des conditions en déplacement (Fig. 1). La zone à soulager est un carré de dimensions $20 \mathrm{~mm} \times 20 \mathrm{~mm}$. Elle est centrée autour du trou.

Le renforcement de la plaque se fait par un patch composite carbone/époxyde dont les plis ont un comportement élastique linéaire orthotrope : $E_{x}=181 \mathrm{GPa}$, $E_{y}=10 \mathrm{GPa}, \nu=0,28, G_{x y}=7 \mathrm{GPa}$. Le patch composite est composé de 6 plis d'épaisseur $0,125 \mathrm{~mm}$ chacun. Le collage entre le patch composite et la plaque d'aluminium est considéré comme parfait : l'épaisseur de la colle est supposée nulle, entraînant une absence de zone de transfert d'efforts entre la plaque et le patch. De ce fait, une modélisation en deux dimensions est choisie.

Le chargement mécanique consiste en un essai de traction modélisé par des déplacements imposés dans la partie supérieure (Fig. 1). La valeur du déplacement imposé $u_{y}$ vaut $1 \mathrm{~mm}$.

L'objectif est de soulager une zone de forme carrée de $20 \mathrm{~mm}$ de coté entourant le trou, avec les contraintes suivantes :

- l'aire du patch composite est limitée à $20 \%$ de l'aire de la plaque d'aluminium,

- le patch ne peut pas recouvrir directement la zone à soulager.

Cette deuxième contrainte est liée à la nécessité de préserver une zone d'accès près du trou pour faciliter la mise en place et l'inspection des systèmes de fixation.

Le code d'éléments finis Ansys 7.0 est utilisé pour résoudre le problème de structure et déterminer la contrainte de Von Mises moyenne dans la zone à renforcer. Des éléments finis $2 \mathrm{D}$ de type plaque à 8 nœuds sont 


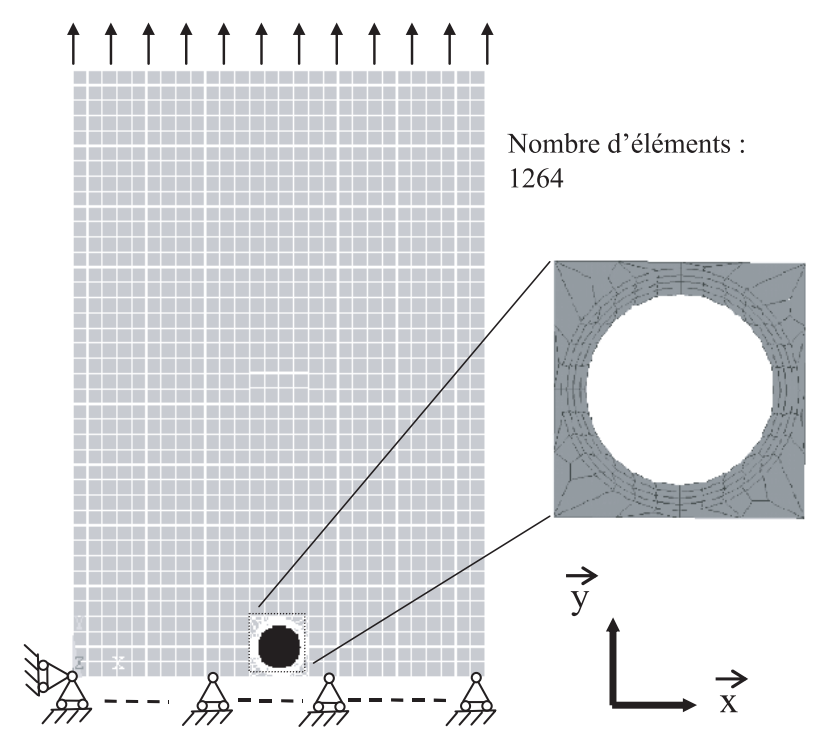

Fig. 2. Maillage de la structure renforcée : la zone à soulager est maillée plus finement.

utilisés afin de modéliser la plaque et le patch composite. Le contact parfait entre le patch et la plaque s'effectue en fusionnant les nœuds. La figure 2 présente le maillage utilisé. Une procédure a été implémentée afin de mailler plus finement au voisinage trou, siège d'un gradient de contraintes important.

Le patch composite a donc pour dimensions $80 \mathrm{~mm} \times$ $80 \mathrm{~mm}$. Ces dimensions sont choisies afin que le maillage du patch corresponde au maillage de la plaque et que l'aire du patch représente environ $20 \%$ de la surface totale de la plaque. Ce patch est centré sur la zone à soulager, en prenant soin de retrancher la zone interdite qui est ici confondue avec la zone à soulager. Les six plis du patch sont tous orientés à $0^{\circ}$ par rapport à la direction de traction $y$ (Fig. 1).

La résolution se fait grâce à une méthode itérative. Une étude de convergence a été effectuée pour garantir la précision du calcul.

\subsection{Analyse du renforcement obtenu avec un patch carré}

Le patch carré a pour effet de renforcer la zone à soulager de 7,1 \% en terme de contrainte de Von Mises moyenne par rapport à la structure sans patch. De par sa position, il renforce « localement » la zone à soulager, mais il ne change pratiquement pas la distribution générale des contraintes loin de ce dernier. Ce renforcement peut être vu simplement comme la conséquence de la présence d'un matériau beaucoup plus rigide près du trou (Fig. 3).

Une autre solution consiste à ne pas se limiter à renforcer localement la structure, mais à dévier de manière forte les contraintes dans la structure. Il serait possible de jouer sur la longueur et la largeur du patch, ainsi que sur sa position sur la structure. Un moyen de définir une grande diversité de géométrie avec peu de points repose sur l'utilisation de courbes splines.
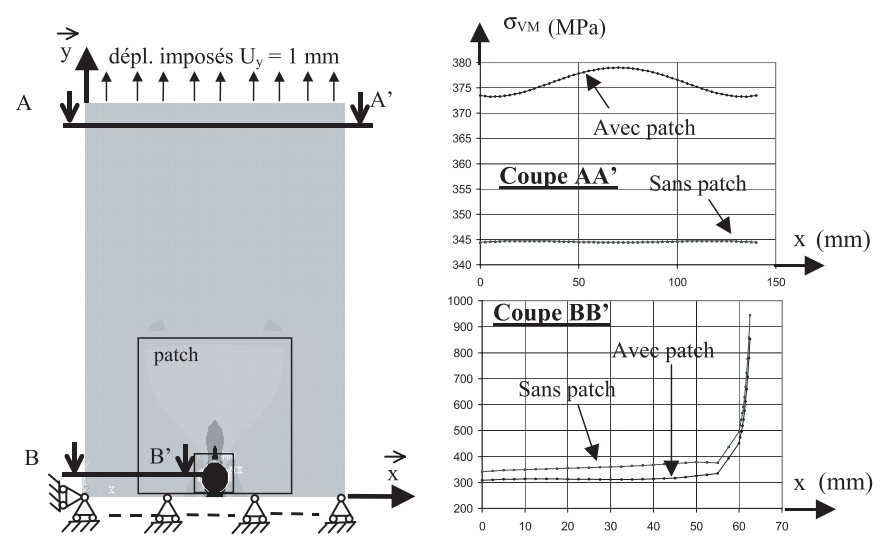

Fig. 3. Analyse du renforcement par un patch de forme carrée.

\section{Optimisation du renfort composite}

\subsection{Modélisation d'un renforcement par un patch de forme complexe}

Une autre solution consiste à ne pas chercher uniquement à renforcer localement la zone endommagée, mais également à dévier les contraintes loin de la zone à renforcer. Un renforcement qui dévierait fortement les contraintes pourrait diminuer davantage les contraintes dans la zone à soulager.

Pour modéliser une famille de patchs de formes suffisamment variées, la géométrie du renfort est définie par une courbe de type spline avec 8 points de contrôle (Fig. 4). Ce nombre est en fait suffisant pour générer une très grande variété de formes.

Le problème de renforcement est supposé bidimensionnel. La forme du patch et sa position dans le repère global de la structure à renforcer sont alors définies par les coordonnées $\left(x_{i}, y_{i}\right)$ des 8 points de contrôle $P_{i}$ de la courbe.

Le matériau composite est constitué de $n$ plis orientés classiquement les uns par rapport aux autres suivant des angles $\theta_{\iota}, \iota=1 \ldots n$, prenant uniquement des valeurs $0^{\circ}$, $-45^{\circ}, 45^{\circ}$ ou $90^{\circ}$. L'orientation globale du composite par rapport au repère global de la structure est définie par un angle $\beta$, définissant l'orientation des plis à $0^{\circ}$ (Fig. 4). Contrairement aux stratifiés optimisés dans un autre cadre que celui du renforcement de structure $[6,7]$, cet angle $\beta$ constitue un degré de liberté qui permet de mieux orienter le patch par rapport à une direction de sollicitation donnée.

Dans un contexte d'optimisation d'un renforcement utilisant un patch composé de 6 plis pour cette première étude, les variables de conception du problème sont placées dans un vecteur $X$ constitué de 23 composantes réparties de la manière suivante :

- 1 composante à valeur réelle pouvant varier de manière continue. Elle décrit l'orientation globale $\beta$ du composite par rapport au repère de la structure,

- 6 composantes prenant des valeurs discrètes dans l'ensemble $\left\{0^{\circ},-45^{\circ}, 45^{\circ}, 90^{\circ}\right\}$. Elles décrivent les orientations des 6 plis du composite par rapport à la direction $x^{\prime}$ définie figure 4 , 


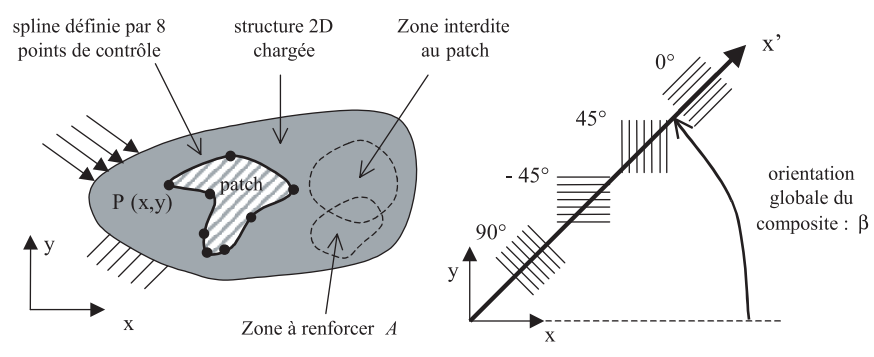

Fig. 4. Modélisation d'un renforcement par un patch composite de forme complexe.

- 16 composantes à valeurs réelles. Elles décrivent les coordonnées des huit points de contrôle de la courbe spline et peuvent varier de façon continue.

L'optimisation présentée dans la section suivante fait intervenir une construction systématique de courbes spline aléatoires. Les courbes splines définissant le périmètre des patchs ne doivent pas former de boucles. À cette fin, une procédure mathématique heuristique originale non décrite ici a été mise en œuvre informatiquement. Cette méthode permet d'obtenir une grande diversité de forme de patch.

L'optimisation nécessite de nombreuses résolutions par éléments finis, pour diverses configurations de renforcement. La géométrie du patch est donc discrétisée de manière similaire pour tous les cas de renforcement en choisissant un maillage de patch superposé à celui de la structure à renforcer.

Après la phase de maillage du patch, les opérations suivantes sont effectuées :

- l'aire du patch est généralement limitée en pratique. Elle est définie dans le présent travail par un pourcentage maximum de couverture de la structure à renforcer égal à $20 \%$. Si cette condition n'est pas vérifiée, le renfort n'est pas considéré,

- des zones de la structure à renforcer ne sont généralement pas accessibles pour des raisons technologiques : passage de vis, réparations... Ces zones interdites peuvent correspondre à la zone à renforcer. Si des éléments finis du patch discrétisé sont présents dans les zones interdites, ils sont simplement éliminés et seul le renfort amputé de cette zone est pris en considération.

\subsection{Optimisation du renforcement par algorithme génétique}

\subsubsection{Principe des algorithmes génétiques}

L'algorithme consiste à simuler l'évolution d'une population de solutions potentielles du problème en s'inspirant des lois de l'évolution naturelle décrites par Darwin au $19^{\mathrm{e}}$ siècle [11].

Le principe a été repris depuis les années 1960 comme outil d'optimisation mathématique. Il s'agit tout d'abord de créer aléatoirement une population initiale d'individus pouvant potentiellement répondre au problème posé.

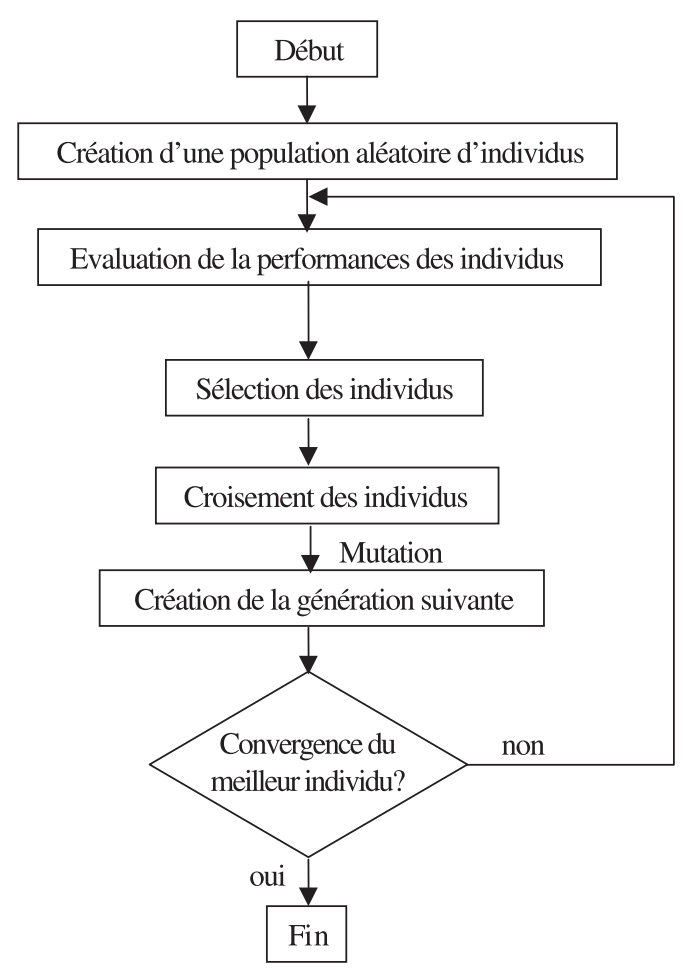

Fig. 5. Algorithme de l'optimisation par algorithme génétique.

Chaque individu est caractérisé par un « chromosome » contenant toutes les variables d'optimisation considérées : dans le présent travail sur le renforcement, il s'agit ici du vecteur $X$ dont les composantes constituent les « gènes » de l'individu. Les individus sont sélectionnés en fonction de leur capacité à répondre au problème posé pour ensuite être croisés et produire alors la génération suivante d'individus (Fig. 5). Le processus est ensuite réitéré en faisant intervenir d'autres opérations comme la mutation, jusqu'à ce que le meilleur individu de la population corresponde à l'optimum du problème posé.

Les méthodes d'optimisation par algorithme génétique sont particulièrement adaptées aux problèmes faisant intervenir des variables discrètes, comme cela est le cas pour l'orientation de plis de composites. De nombreux exemples d'optimisation de structures composites stratifiées sont disponibles dans la littérature $([6,7]$ par exemple), ce qui montre le bien-fondé de ce type d'approche.

\subsection{2 Évaluation des individus}

Les individus sont évalués en fonction de leur capacité à renforcer la structure. Pour cette première approche, la fonction objectif $F O$ est ici simplement représentée par la moyenne des contraintes de Von Mises $\sigma_{\text {Von Mises }}$ dans la zone $A$ à renforcer de la structure (Fig. 4) :

$$
F O=\frac{1}{\operatorname{Aire}(A)} \int_{A} \sigma_{\text {Von Mises }} \mathrm{d} s
$$


Parent 1

\begin{tabular}{|c|c|c|c|c|c|}
\hline$-45^{\circ}$ & $0^{\circ}$ & $45^{\circ}$ & $90^{\circ}$ & $-45^{\circ}$ & $0^{\circ}$ \\
\hline
\end{tabular}

Parent 2

\begin{tabular}{|c|c|c|c|c|c|}
\hline $0^{\circ}$ & $-45^{\circ}$ & $90^{\circ}$ & $-45^{\circ}$ & $90^{\circ}$ & $-45^{\circ}$ \\
\hline
\end{tabular}

Enfant 1

\begin{tabular}{|c|c|c|c|c|c|}
\hline$-45^{\circ}$ & $0^{\circ}$ & $90^{\circ}$ & $-45^{\circ}$ & $-45^{\circ}$ & $0^{\circ}$ \\
\hline
\end{tabular}

Enfant 2

\begin{tabular}{|c|c|c|c|c|c|}
\hline $0^{\circ}$ & $-45^{\circ}$ & $45^{\circ}$ & $90^{\circ}$ & $0^{\circ}$ & $-45^{\circ}$ \\
\hline pli 1 & pli 2 & pli 3 & pli 4 & \multicolumn{1}{c}{ pli 5} & pli 6
\end{tabular}

Mutation du pli de $90^{\circ}$ à $0^{\circ}$

Fig. 6. Illustration des opérations de croisement binaire et de mutation binaire pour les gènes associés aux orientations d'un composite à 6 plis.

À chaque génération, la fonction coût $F_{i}$ d'un individu $i$ est définie comme la différence entre la fonction objectif du plus mauvais individu de la population et sa fonction objectif pour avoir un classement décroissant du meilleur individu au plus mauvais :

$$
F_{i}=F O_{(\text {plus mauvais individu })}-F O_{i}
$$

La probabilité de survie $P_{i}$ d'un individu $i$ d'une population constituée de $N I$ individus est alors définie à partir de sa fonction coût :

$$
P_{i}=\frac{F_{i}}{\sum_{j=1}^{N I} F_{j}}
$$

Des tirages aléatoires sont ensuite réalisés pour décider de la survie ou non de chaque individu. Bien que les meilleurs individus soient favorisés, cette procédure donne une chance à toute configuration de renforcement.

\subsubsection{Croisement des individus et mutation des gènes}

Les opérations de croisement consistent à produire de nouveaux individus dont les caractéristiques sont héritées des parents sélectionnés dans la génération précédente. Une distinction est faite entre les variables discrètes et réelles du problème.

La figure 6 illustre une opération de croisement des gènes décrivant l'orientation de six plis avec un nombre de point de croisement égal à trois. Une mutation est également présentée sur cette figure : elle est définie par une probabilité de mutation $P_{M}$ du gène puis, le cas échéant, par la modification aléatoire de la valeur du gène. Cette procédure permet constamment d'altérer la valeur des gènes pour éventuellement produire des individus performants que de simples croisements ne produiraient pas.

Le cas des variables réelles fait intervenir une proportion des gènes respectifs des parents. Pour chaque croisement, une variable $w$ est obtenue aléatoirement dans l'intervalle $[0,1]$ et s'applique de manière similaire pour tous les gènes réels du chromosome :

Composantes $(x, y)$ des points de contrôle de la spline :

$$
\begin{aligned}
& x_{\text {enfant }}=w x_{\text {parent 1 }}+(1-w) x_{\text {parent 2 }} \\
& y_{\text {enfant }}=w y_{\text {parent 1 }}+(1-w) y_{\text {parent 2 }}
\end{aligned}
$$

Orientation $\beta$ du composite :

$$
\beta_{\text {enfant }}=w \beta_{\text {parent } 1}+(1-w) \beta_{\text {parent } 2}
$$

Une probabilité de mutation réelle est également choisie afin d'altérer la valeur des gènes réels et produire éventuellement des enfants performants. Elle est généralement très petite.

De manière à favoriser la convergence de l'algorithme, le meilleur individu de chaque génération est automatiquement cloné dans la génération suivante. L'algorithme est alors qualifié d'élitiste. Pour accélérer la convergence, de faibles modifications du chromosome X du meilleur individu sont également testées à chaque génération pour tenter de repérer de meilleurs individus ; ce processus, appelé « croisement primitif », s'apparente à une démarche d'optimisation locale par algorithme de descente.

\section{Application}

\subsection{Résultat}

L'algorithme génétique est utilisé pour optimiser le patch composite du problème de renforcement de structure présenté (Fig. 1). Le calcul a été mené sur un processeur $3 \mathrm{GHz}$ et a duré 2 jours. La courbe 7 représente la courbe de convergence de l'algorithme génétique : la valeur de la contrainte moyenne de Von Mises de la meilleure solution de chaque population est représentée en fonction du nombre d'itérations. La meilleure solution trouvée a été atteinte au bout de 45 générations. L'algorithme comporte trois phases d'évolution principales. Durant les premières générations, une évolution rapide du meilleur individu est observée; une évolution plus lente se produit ensuite et correspond en partie à l'utilisation du croisement primitif; finalement, le meilleur individu 


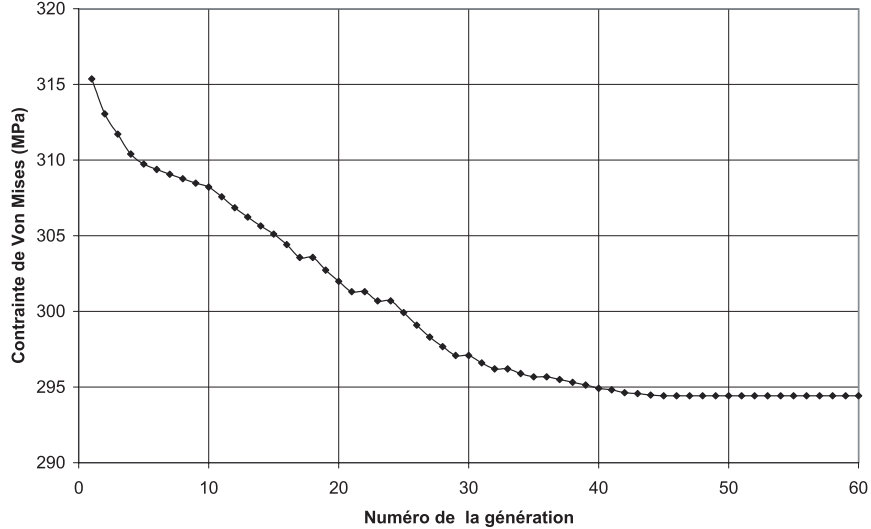

Fig. 7. Évolution la contrainte de Von Mises de la meilleure solution en fonction du nombre d'itérations.

reste identique pour les dernières générations. Il est supposé correspondre à la solution optimale du problème de renforcement.

La figure 8 représente la forme du patch obtenu et la contrainte de Von Mises dans la plaque. Dans l'exemple donné, les angles des plis sont tous orientés à $-1^{\circ}$ par rapport à l'axe des $y$. Le patch renforce de $17,2 \%$ la zone à soulager, ce qui est à comparer avec les $7,1 \%$ lors de l'utilisation du patch carré (Sect. 2.3).

\subsection{Interprétation}

Le renforcement est en fait dû à deux phénomènes distincts :

- un renforcement local provoqué par la partie du patch qui entoure le trou,

- un renforcement global lié à la forme allongée du patch, entraînant une redistribution forte des contraintes dans la structure.

Le renforcement principal est obtenu par l'effet global. En effet, le patch carré précédemment présenté ne soulage que localement et ce soulagement n'atteint que $7,1 \%$. Le phénomène de renforcement global est obtenu par la forme allongée du patch. La figure 9 représente le champ de contraintes de Von Mises dans la plaque. La dissymétrie ainsi que l'anisotropie du patch optimisé a pour effet d'orienter principalement le flux des contraintes dans la partie droite de la plaque. Le patch soulage donc le reste de la structure.

La contrainte maximale de Von Mises atteinte dans la zone à soulager a également diminué de $17 \%$. Les contraintes passent principalement dans la partie droite de la structure (Fig. 8, coupe A-A'). Une surcontrainte apparaît en bordure de patch à droite de la zone à soulager (Fig. 8, coupe B-B').

Il faut souligner qu'une légère variation de la direction des plis ou des points de contrôle ne change que très faiblement les résultats : la solution proposée par l'outil d'optimisation présenté peut donc être considérée comme stable. Notons enfin que la solution obtenue n'est pas

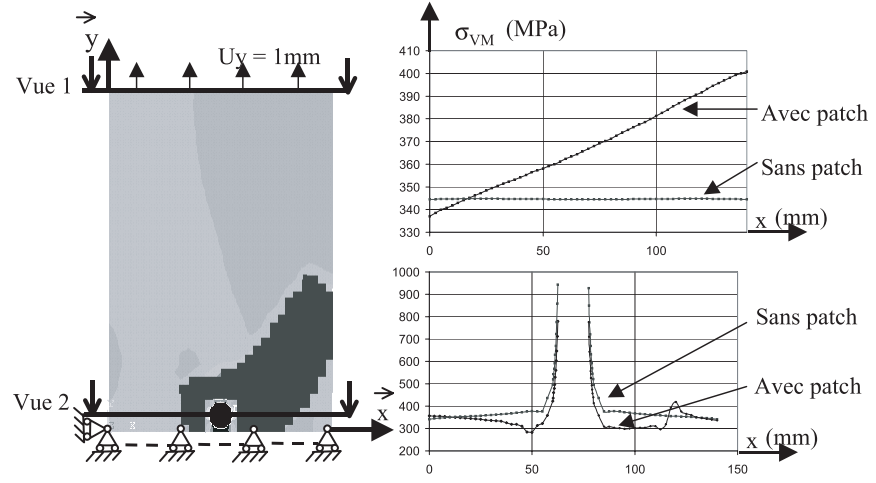

Fig. 8. Géométrie du patch optimale et contrainte de Von Mises dans la plaque.

analytiquement calculable, ce qui souligne l'intérêt de la présente démarche.

\section{Conclusion}

Cet article décrit le rôle préventif d'un renforcement par patch composite sur les structures vieillissantes. Un outil de modélisation du renforcement a été présenté. Il utilise un code de calcul par éléments finis. Le renforcement local d'un patch de forme carrée a été modélisé. Cette première approche s'est poursuivie par la prise en considération de géométrie de patchs plus complexes afin d'obtenir un renforcement plus important. L'utilisation de courbes splines permet d'avoir une grande diversité de patchs avec peu de points définissant sa géométrie. L'outil d'optimisation choisi est un algorithme génétique.

Dans le cas d'un essai de traction sur une plaque trouée, la configuration de patch optimale met en évidence deux aspects du renforcement : un phénomène de renforcement local lié à la présence du patch près de la zone à soulager et un phénomène de renforcement global consistant en une redistribution générale des contraintes dans la structure entière.

Un prolongement de ce travail est la prise en compte de critères mécaniques appropriés à un renforcement de structure soumise à des phénomènes de fatigue et à des chargements plus complexes.

\section{Références}

[1] A. Baker, Repair of Cracked or Defective Metalic Aircraft Components with Advanced Fibre Composites - An Overview of Australian Work, Composite structure 2 (1984) 153-181

[2] A. Baker, Bonded composite repair of metallic aircraft components - Overview of australian activities, AGARDCP-550 (1995) 1-14

[3] A. Baker, Bonded composite repair of fatigue-cracked primary aircraft structure, Composite structure 47 (1999) 431-443

[4] V. Kradinov, J. Hanauska, A. Barut, E. Madenci, D.R. Ambur, Bolted patch repair of composite panels with a cutout, Composite structure 56 (2002) 423-444 
[5] Z.P. Marioli-Riga, G.J. Tsamasphyros, G.N. Kanderakis, Design of emergency aircraft repairs using composite patchs, Mechanics of composite materials and structures 8 (2001) 199-204

[6] R. Le Riche, Optimization of composite structures by genetic algorithms, Thèse, Virginia institute and state university, 1994

[7] R. Le Riche, R.T. Haftka, Improved genetic algorithm for minimum thickness composite laminate design, Composites Engineering 5 (1995) 143-161

[8] Chung Ki-Hyun, Yang Won-Ho, A study on the fatigue crack growth behavior of thick aluminum panels repaired with a composite patch, Composite Structures 60 (2003) $1-7$

[9] G.J. Tsamasphyros, G.N. Kanderakis, D. Karalekas, D. Rapti, E.E. Gdoutos, D. Zacharopoulos, Z.P. MarioliRiga, Study of composite patch repair by analytical and numerical methods, Fatigue Fracture Engineering Material Structure 24 (2001) 631-636

[10] Seo Dae-Cheol, Lee Jung-Ju, Fatigue crack growth behavior of cracked aluminum plate repaired with composite patch, Composite Structures 57 (2002) 323-330

[11] Darwin Charles, The origin of species, http://etext.library.adelaide.edu.au/d/d22o/, 1859 